\title{
Measurements of Higgs boson production using decays to two $b$-quarks with the ATLAS detector
}

\author{
Rute Pedro* ${ }^{\dagger}$, on behalf of the ATLAS Collaboration \\ LIP - Laboratório de Instrumentação e Física Experimental de Partículas, Portugal \\ E-mail: rute@lip.pt, rute.pedro@cern.ch
}

\begin{abstract}
Testing the large coupling of the Higgs boson to $b$-quarks is particularly relevant in order to probe the Higgs Sector, providing at the same time an important input to many new physics searches with final states containing this Higgs decay. These proceedings present the first results of the observation of $H \rightarrow b b$ decays and further measurements involving this decay channel, using data from the LHC proton-proton collisions at a center-of-mass energy of $\sqrt{s}=13 \mathrm{TeV}$. The differential cross-section of Higgs production in association with a vector boson was measured in the context of the simplified template cross-section and is presented here along with an interpretation based on the effective field theory framework. Moreover, an inclusive search for large momentum $H \rightarrow b b$ decays is summarised.
\end{abstract}

European Physical Society Conference on High Energy Physics - EPS-HEP2019 -

10-17 July, 2019

Ghent, Belgium

\footnotetext{
* Speaker.

${ }^{\dagger}$ The author acknowledges the financial support of Fundação para a Ciência e a Tecnologia (FCT) through the project PTDC/FIS-PAR/29147/2017.
} 


\section{Introduction}

Measurements of Higgs boson production using decays to two $b$-quarks with the ATLAS detector [1] are summarised. With a branching ratio of $58 \%$ for $m_{H}=125 \mathrm{GeV}$, the $H \rightarrow b b$ decay is the dominant one in the Standard Model (SM) and drives the Higgs total width, a quantity to which the LHC experiments have very low sensitivity. Measurements of this process allows the Higgs couplings to be further constrained and to place limits on the branching fraction allowed for possible exotic decays.

Inclusive searches are disfavoured due to the large QCD background, but signal sensitivity is recovered in the regime where the Higgs is produced with high momentum, as presented in Section 5. However, the golden channel to perform $H \rightarrow b b$ studies is via vector boson associated production, $V H$, with $V=W, Z$. The leptonic decay of the vector boson provides a good trigger and efficiently reduces competing backgrounds. This analysis is presented in Section 2 for the LHC Run 2 data set. The results, shown in Section 3, are combined with other final states and with Run 1 data, yielding the observation of $H \rightarrow b b$ decays and $V H$ production. Furthermore, Section 4 presents the $V H$ cross-section $\left(\sigma_{V H}\right)$ measurements in the context of the Simplified Template Cross Section (STXS) and their interpretation in the context of Effective Field Theory (EFT).

\section{2. $V H(H \rightarrow b b)$ analysis}

The analysis is designed to reconstruct the leptonic decays of the vector boson, in three channels: 0-lepton $(Z \rightarrow v v)$, 1-lepton $(W \rightarrow l v)$ and 2-leptons $(Z \rightarrow l l)$, where lepton here designates an electron or a muon [2]. The 0-lepton channel employs a missing transverse energy (MET) trigger and events with MET $>150 \mathrm{GeV}$ are selected offline. Events in the 1-lepton channel pass single electron or MET triggers. Offline, the events are required to have 1 isolated electron or muon with $p_{\mathrm{T}}>27$ or $25 \mathrm{GeV}$, respectively. The 2-lepton channel also uses single lepton triggers and requires 2 electrons or muons. The $H \rightarrow b b$ candidate is selected requiring 2 (or 3) final state jets, built from calorimeter energy clusters grouped using the jet finding algorithm anti- $k_{t}$ with radius parameter 0.4. The two leading jets should be identified as originating from $b$-quark fragmentation. This is achieved with the b-tagging algorithm MV2, that gathers information from the tracking system (such as track displacement and the existence of multiple vertices) to build a multivariate discriminant.

The distributions of the MET, transverse mass of the reconstructed $W$, and the invariant mass of the lepton pair are presented in Figure 1 for the 0-,1- and 2-lepton channels. The background contribution is obtained from Monte Carlo simulation: $Z+$ jets dominates in the 0- and 2-lepton channels, while top pair production $(t \bar{t})$ and $W+$ jets are the main backgrounds in the 1-lepton case. A sample pure in $t \bar{t}$ is obtained by using 2-lepton events with different flavour leptons $(e \mu)$, allowing to constrain this important background with collision data. Similarly, a control region for the $W+$ heavy flavour (HF) jets background is defined in the 1-lepton channel ${ }^{1}$.

A Boosted Decision Tree (BDT) is employed to improve the sensitivity to the signal, as shown in Figure 2. The data are analysed with a profile likelihood binned fit of the simulation of the BDT

\footnotetext{
${ }^{1}$ By selecting events for which the invariant mass of the $b b$ system is incompatible with the SM Higgs mass $\left(m_{b b}\right.$ $<75 \mathrm{GeV}$ ), and for which the system composed of $\ell v b$ has mass well above the SM top value: $m_{\text {top }}>225 \mathrm{GeV}$.
} 

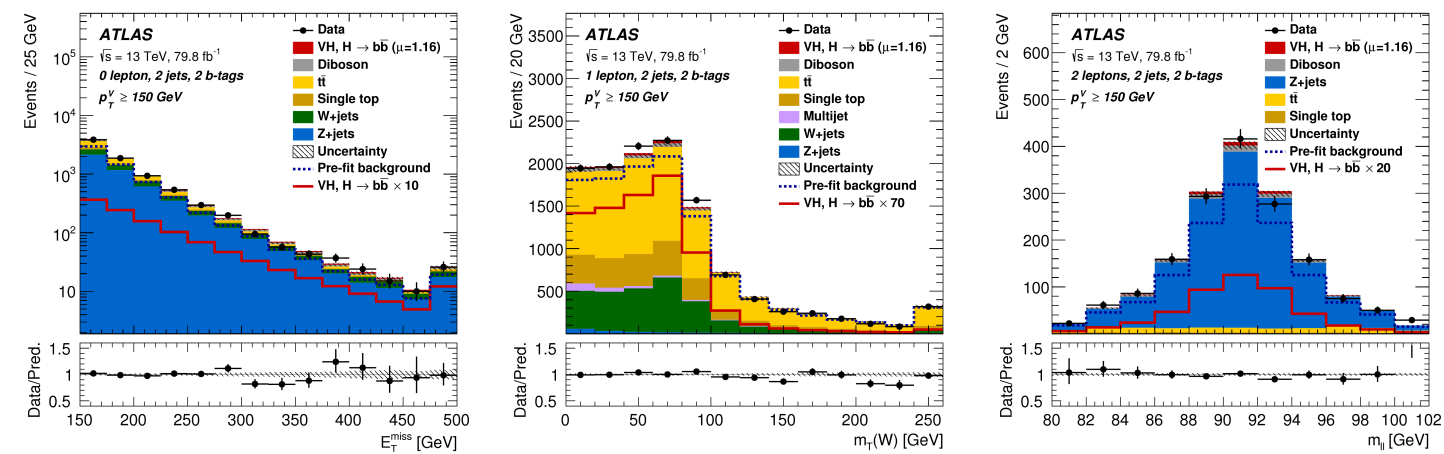

Figure 1: Distribution of the MET, $W$ candidate $p_{\mathrm{T}}$ and invariant mass of the 2 lepton system in the (left) $0-$, (middle) 1- and (right) 2-lepton channels, respectively [2].
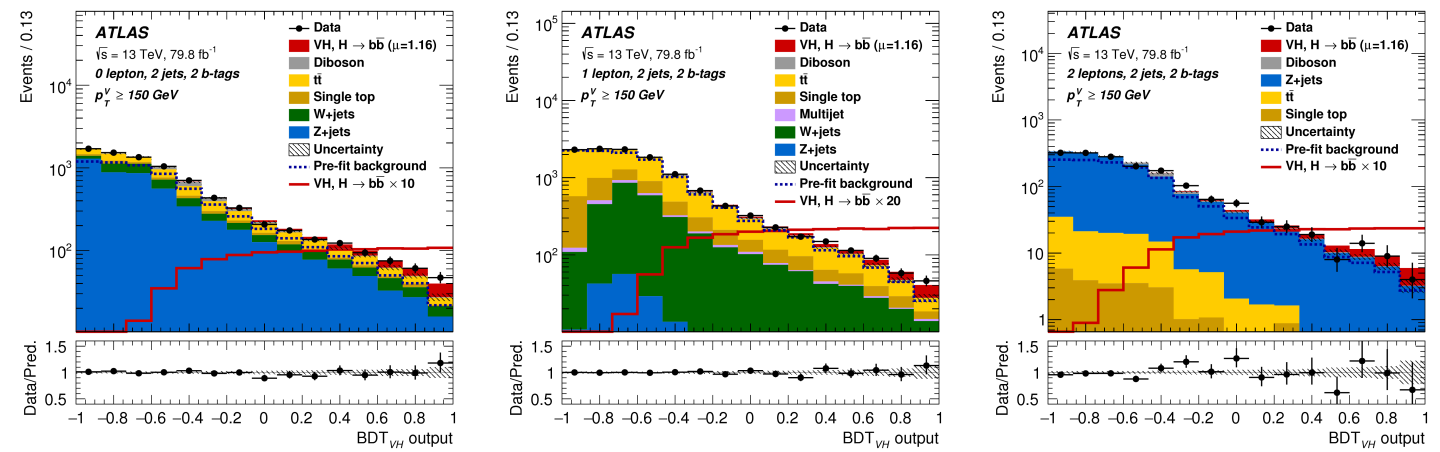

Figure 2: Distribution of the BDT output in the (left) 0-, (middle) 1- and (right) 2-lepton channels [2].

distribution in the signal regions, and the $m_{b b}$ distribution (event yield) in the top- $e \mu$ ( $W+\mathrm{HF}$ jets) control regions. The scales of the main backgrounds are determined from data and the uncertainties of the analysis are implemented as fit nuisance parameters.

A total integrated luminosity of $79.8 \mathrm{fb}^{-1}$ of $\sqrt{s}=13 \mathrm{TeV}$ proton-proton collision data were analysed. The signal significance observed in data reaches up to $5 \sigma$ above the expected background, as can be seen in Figure 3. The measured signal strength was $\mu=1.16_{-0.25}^{+0.27}$ and the corresponding observed (expected) significance was $4.9(4.3) \sigma$. The results are cross-checked with a cut-based analysis yielding compatible signal measurements. Figure 3 also presents the respective signal peak subtracted from background.

\section{Observation of $H \rightarrow b b$ decays and $V H$ production}

The $H \rightarrow b b$ signal is observed by combining the Run 1 and Run $2 V H$ analyses results, and $H \rightarrow b b$ searches using different Higgs production mechanisms, namely: $t \bar{t} H$, vector boson fusion (VBF), and gluon fusion ( $\mathrm{ggF}$ ) [2]. The significance reaches 5.4 $\sigma$. Figure 4 shows the signal strength measurement, and the impact of each final state in the combined result, with $V H$ production standing out as the most precise. Conversely, $H \rightarrow b b$ is the dominant contribution to the analysis that led to the $5.3 \sigma$ observation of $V H$ production, involving the combination with the $H \rightarrow \gamma \gamma$ and $H \rightarrow Z Z \rightarrow 4 l$ final states, as shown in Figure 4. The $W H$ and $Z H$ modes are 

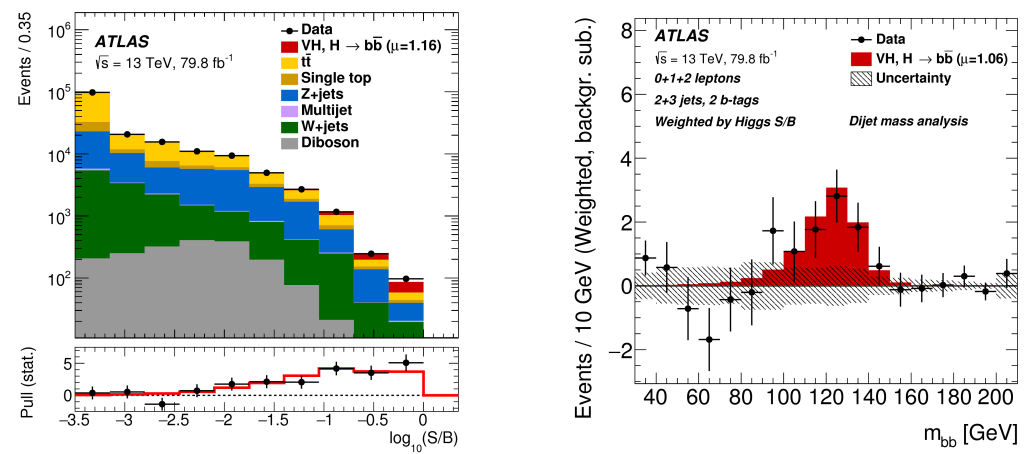

Figure 3: (Left) Combined post-fit event yields in bins of signal significance $\left(\log _{10}(S / B)\right)$, and expected/observed deviation of data with respect to the background-only scenario (in standard deviation units $(\sigma))$. (Right) Background-subtracted post-fit distribution of $m_{b b}$ in the cut-based analysis [2].
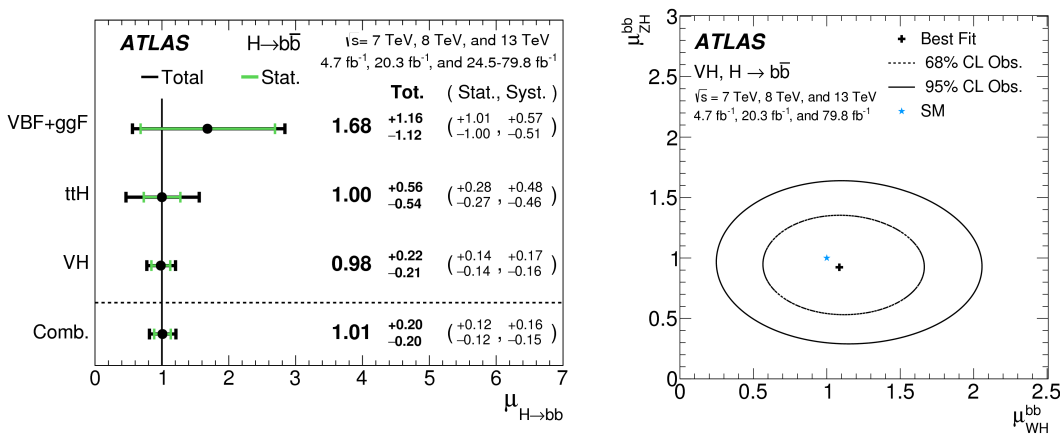

\begin{tabular}{lcc}
\hline \multirow{2}{*}{ Channel } & \multicolumn{2}{c}{ Significance } \\
\cline { 2 - 3 } & Exp. & Obs. \\
\hline$H \rightarrow Z Z^{*} \rightarrow 4 \ell$ & 1.1 & 1.1 \\
$H \rightarrow \gamma \gamma$ & 1.9 & 1.9 \\
$H \rightarrow b \bar{b}$ & 4.3 & 4.9 \\
\hline VH combined & 4.8 & 5.3 \\
\hline
\end{tabular}

Figure 4: (Left) Measured $H \rightarrow b b$ signal strength split by the different final states (VBF+ggF, $t \bar{t} H$ and $V H$ ) and combination. (Middle) Signal strength separated by $W H$ and $Z H$ production. (Right) Observed and expected significances of the $V H$ production in the different final states and combining all final states [2].

separately measured in the dominant decay channel. All measurements are compatible with the SM prediction, within the limits of their uncertainties.

\section{Measurement of the $V H$ cross section in the STXS and EFT interpretaion}

The $V H(H \rightarrow b b)$ process was used to measure the $V H$ differential cross-section $\left(\sigma_{V H}\right)$ within the STXS framework [3]. This framework is designed to probe the Higgs cross-section $\sigma_{H}$ in the various production modes, using exclusive regions of phase space for which the granularity is increased as more collision data is acquired and statistics allows. For $V H$, the measurement is a function of the vector boson transverse momentum $\left(p_{\mathrm{T}}^{V}\right)$, and the $W H$ and $Z H$ modes are separated.

The acceptance of the analysis regions to the STXS bins is presented in Figure 5. The crosssection $\sigma_{V H}$ is measured for each STXS bin by fitting the corresponding signal template, exemplified in Figure 5, to the BDT output distribution in data. ${ }^{2}$ Figure 6 shows the results, the SM prediction, and the good agreement between the two.

\footnotetext{
${ }^{2}$ The lower $p_{\mathrm{T}}^{V}$ bins are not measured due to the poor acceptance.
} 

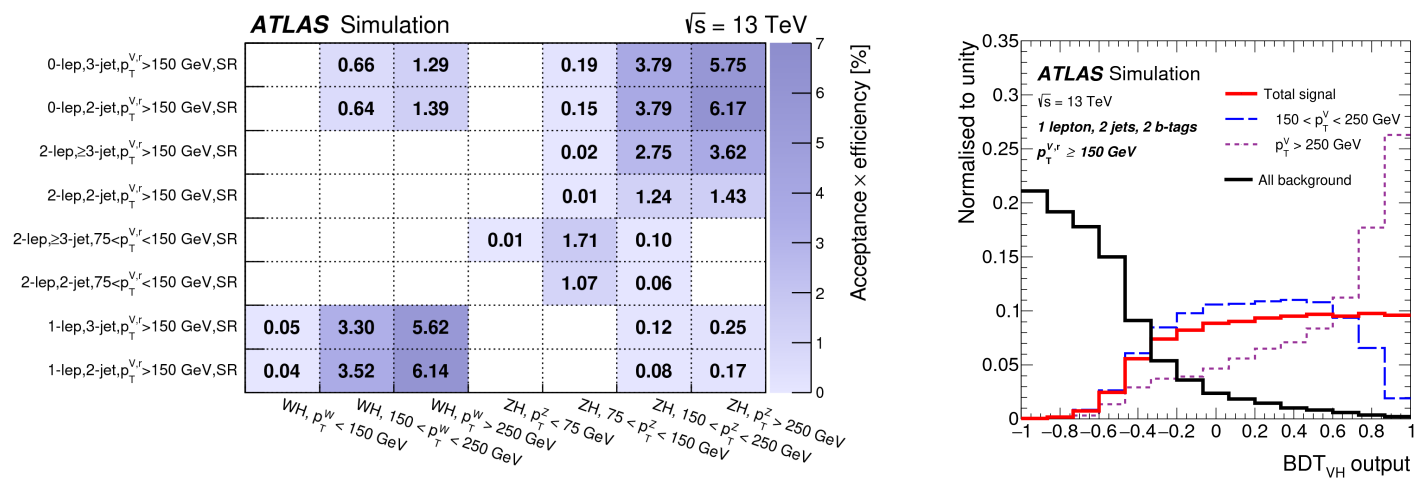

Figure 5: (Left) Acceptance of the analysis regions ( $y$-axis) to the STXS bins ( $x$-axis). (Right) Background and signal BDT distribution, and simulated template of the BDT distribution for two STXS bins [3].
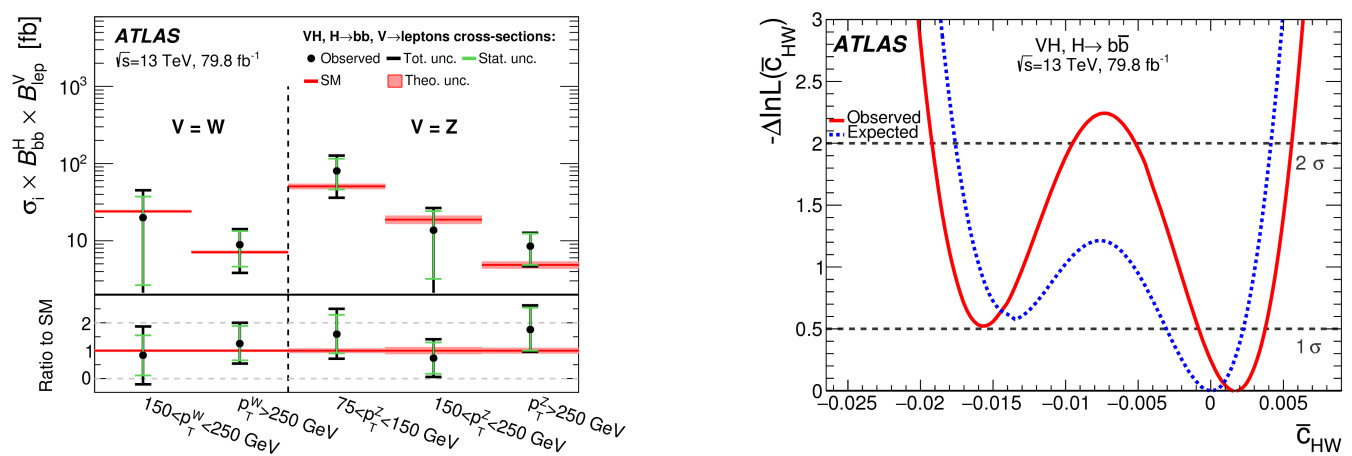

Figure 6: (Left) Measured and predicted $\sigma_{V H}$ in the various STXS bins. (Right) Expected and observed limits on the $c_{H W}$ EFT coefficient [3].

These results are then interpreted in the context of EFT to investigate the $H V V$ coupling vertex [3]. Broadly speaking, the EFT framework adds extra terms to the SM Lagrangian to model possible anomalous Higgs couplings. Then, Higgs measurements are used to place constraints on the strength $c_{i}$ of the new interaction terms. Recasting the STXS results, the $c_{H W}$ and $c_{W}-c_{B}$ are constrained to few percent, at 95\% confidence level (CL), shown in Figure 6 for the case of $c_{H W}$. These two coefficients are 0 if the $H V V$ coupling is SM-like. Otherwise, the $\sigma_{W H / Z H}$ is affected by contributions resulting from the EFT extra terms.

\section{Inclusive search for $H \rightarrow b b$ in the high momentum regime}

New physics is more expected to be revealed at high energies, where the Higgs is produced with a large boost. In this regime, the leading order signature of $H \rightarrow b b$ is characterised by a large radius jet with two sub- $b$-jets.

ATLAS performed an inclusive search for boosted resonances, where the $H \rightarrow b b$ decay is included [4]. The signal is reconstructed as a large radius $\mathrm{R}=1.0$ jet with $p_{\mathrm{T}}>480 \mathrm{GeV}$, recoiling against a second large radius jet. The invariant mass of the signal-candidate jet is analysed in the range between 70 and $230 \mathrm{GeV}$. To improve the resolution, jets are trimmed and their mass is 

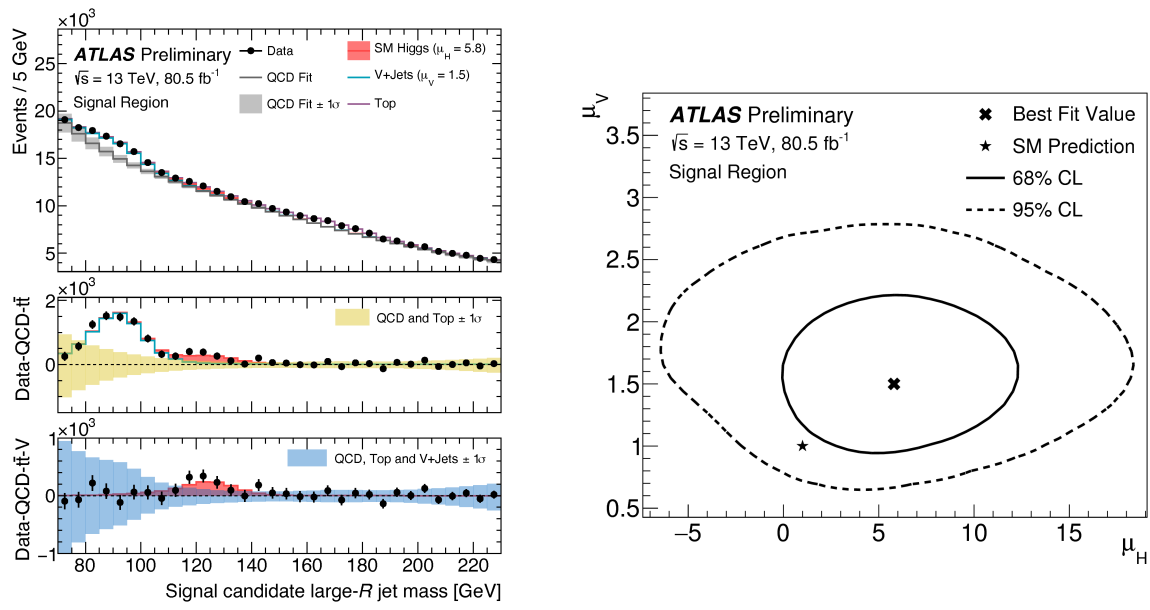

Figure 7: (Left) Post-fit distribution of the signal candidate large radius jet mass including all backgrounds and successively removing the QCD, top and $V+$ jets backgrounds. (Right) Signal strength of the $H+$ jets $\left(\mu_{H}\right)$ and $V+$ jets $\left(\mu_{V}\right)$ processes [4].

calibrated combining information from the tracking detector. The backgrounds are composed of $W / Z+$ jets and top. The dominant QCD background is determined with data-driven methods. The post-fit distribution of the leading jet mass, obtained by analysing $80.5 \mathrm{fb}^{-1}$ of data, are presented in Figure 7. As a cross-check, the analysis includes a $5 \sigma$ measurement of $V+$ jets production. For the boosted $H+$ jets, the observed signal significance is $1.6 \sigma$. Figure 7 shows the signal strength of the two processes, both compatible with the SM prediction within the uncertainties.

\section{Summary}

The $H \rightarrow b b$ decays were used in a prolific set of measurements with the ATLAS experiment at the LHC. First, the observation of $H \rightarrow b b$ with a signal strength of $\mu=1.01 \pm 0.20$ confirmed the Higgs coupling to the down-type quark sector. Then, the $V H$ differential cross-section was measured in bins of the STXS. The results are compatible with the SM within the uncertainties, which are largely dominated by the data statistics. These results were recast in terms of limits to additional effective theory operators, possibly affecting the $H V V$ interaction vertex: $c_{H W}$ and $c_{W}-c_{B}$ are limited to few percent at 95\% CL. Finally, an inclusive search for $H \rightarrow b b$ in the high momentum regime was performed, reaching an observed signal significance level of $1.6 \sigma$.

\section{References}

[1] ATLAS Collaboration, The ATLAS Experiment at the CERN Large Hadron Collider, JINST 3 (2008) S08003

[2] ATLAS Collaboration, Observation of $H \rightarrow b \bar{b}$ decays and $V H$ production with the ATLAS detector, Phys. Lett. B786 (2018) 59-86

[3] ATLAS Collaboration, Measurement of $V H, H \rightarrow b \bar{b}$ production as a function of the vector-boson transverse momentum in $13 \mathrm{TeV}$ pp collisions with the ATLAS detector, JHEP 1905 (2019) 141

[4] ATLAS Collaboration, Search for boosted resonances decaying to two b-quarks and produced in association with a jet at $\sqrt{s}=13 \mathrm{TeV}$ with the ATLAS detector, ATLAS-CONF-2018-052 